\title{
Studying the early solar system: exploration of minor bodies with spaceborne VIS/IR spectrometers: a review and prospects
}

Gabriele E. Arnold, J. Helbert, D. Kappel

Gabriele E. Arnold, J. Helbert, D. Kappel, "Studying the early solar system: exploration of minor bodies with spaceborne VIS/IR spectrometers: a review and prospects," Proc. SPIE 10765, Infrared Remote Sensing and Instrumentation XXVI, 1076508 (18 September 2018); doi: $10.1117 / 12.2320667$

SPIE Event: SPIE Optical Engineering + Applications, 2018, San Diego, California, United States 


\title{
Studying the early solar system - exploration of minor bodies with spaceborne VIS/IR spectrometers: a review and prospects
}

\author{
Gabriele E. Arnold*, J. Helbert, D. Kappel \\ Deutsches Zentrum für Luft- und Raumfahrt e.V. (DLR), Institute of Planetary Research, \\ Rutherfordstrasse 2, 12489 Berlin, Germany. \\ *Gabriele.Arnold@dlr.de; phone +49-3067055370; fax + 49-3067055303
}

\begin{abstract}
Small bodies like asteroids and comets are little differentiated objects that have preserved information about the early state of our solar system. Depending on the heliocentric distance of their origin and their further development they exhibit different pristine compositions that include minerals, ices, and refractory organics. Thus, the composition analysis of minor bodies enables investigations of their evolutionary paths and insights into early processes of planetary formation. In the last decades, new spaceflight and instrument technologies enabled detailed investigations of such bodies during flybys, orbital observations, in situ studies with descent probes, and lander experiments. Core payload elements of these missions include VIS to IR spectrometers, which provided regional and global maps of surface composition, texture, and temperature. The wide range of materials to be detected and the large variability of measurement conditions require dedicated instrumentation and observation strategies for this purpose. This paper reviews the state of the art knowledge and technology achievements in this research field. Scientific results, requirements, and instrumental solutions for minor planetary body studies with VIS to IR spectrometers and radiometers are discussed using examples like the Rosetta mission to comet 67P/Churyumov-Gerasimenko, the asteroid missions Dawn to 4 Vesta and 1 Ceres (dwarf planet), and Hayabusa2 to 162173 Ryugu. Based on our present knowledge, open questions in minor body research are summarized, and resulting scientific and instrumental requirements for future spaceborne VIS/IR spectral studies are elaborated in detail.
\end{abstract}

Keywords: Planetary remote sensing, minor planetary bodies, VIS/IR spectroscopy, early planetary evolution processes.

\section{INTRODUCTION}

In September 2016, the ESA mission Rosetta ended at comet 67P/ Churyumov-Gerasimenko, henceforth shortly called $67 \mathrm{P}^{1}$, with a landing of the Rosetta orbiter on the comet more than a year after 67P's perihelion passage. This mission opened a new chapter in the exploration of small bodies in the solar system with space experiments. Rosetta was the first deep space mission to orbit a comet and escorting it during its passage through the inner solar system ${ }^{2,3,4,5,6}$. It became the first one to land a robotic device, "Philae", on a cometary surface in November 2014 ${ }^{7}$ Eleven orbital ${ }^{8}$ and ten lander instruments ${ }^{9}$ collected unique data during the term of Rosetta's operation. In addition, a unique mission profile covering close-up pre-, post- and perihelion observations significantly increased our knowledge about 67P and comets in general. Among the experiments on the orbiter of the Rosetta mission was an imaging VIS/NIR spectrometer VIRTIS (Visible and Infrared Thermal Imaging Spectrometer) to study the comet's surface and coma composition, its surface temperature and texture as well as dynamic processes and the sources of cometary activity ${ }^{10,11}$. VIRTIS is a spaceborne planetary imaging spectrometer technique whose beginnings can be traced back to experiments like OMEGA on Mars Express (ESA) ${ }^{12}$ and which war continued by instruments such as VIRTIS on Venus Express (ESA) ${ }^{13},{ }^{14}$, VIR (Visible and IR Spectrometer) on Dawn (NASA) ${ }^{15}$, and MAJIS (Moons and Jupiter Imaging Spectrometer) ${ }^{16}$ that is currently being developed for the Jupiter Icy moons Explorer (JUICE, ESA). Spectrometer designs for VIS to NIR spectral studies of comets like 67P and low albedo asteroids are driven by high signal-to-noise performances. In addition, most of these objects often display featureless red-slope VIS spectra, which complicate their surface composition analysis within this 
spectral range. This concerns in particular the detection and investigation minerals which could occur. In contrast, the NIR spectral range is well suited for the investigation of water ice deposits and refractory organic materials; whereas the TIR spectral range would be appropriate to detect minerals including iron-poor species. Therefore, new developments are being driven forward which provide for the combined use of VIS/NIR and TIR spectrometers in order to comprehensively examine and map the compositional diversity of these objects. This is the prerequisite for a qualified discussion that would allow essential conclusions to be drawn about the formation and evolution of the objects. The present paper summarizes some results of spaceborne spectral VIS/NIR studies and derives requirements, prospects, and strategies for future spectral investigations of small bodies.

\section{EXAMPLES OF SPACEBORNE SPECTRAL STUDIES OF MINOR BODIES}

Minor bodies like asteroids and dwarf planets visited by spacecraft are 951 Gaspra, 243 Ida (Galileo flybys), 253 Mathilde, 433 Eros (NEAR Shoemaker), 9969 Braille (Deep Space 1), 5535 Annefrank, 25143 Itokawa (Hayabusa), 2867 Śteins (Rosetta), 21 Lutetia (Rosetta), 4 Vesta (Dawn), 4179 Toutatis (Chang's 2), 1 Ceres (Dawn, ongoing), 134340 Pluto (New Horizons), and 162173 Ryugy (Hayabusa2, ongoing). Comets studied with spacecraft are 21P/Giacobini-Zinner (ICE), 1P/Halley (Vega 1, Vega 2, Suisei, Sakigake, Giotto), 26P/Grigg-Skjellerup (Giotto), 19P/Borrelly (Deep Space 1), 81P/Wild 2 (Stardust), 9P/Tempel 1 (Deep Impact, Stardust), C/2006 P 1 (Ulysses), 103P/Hartley 2 (EPOXI), and 67P (Rosetta). Most of these space missions were equipped with radiometers and/or spectrometers which are not all discussed in this paper. This paper focuses on the more recent missions Rosetta and Dawn only. Section 2 discusses some scientific result of spectral measurements. They are dedicated to show the capacities and limits of current investigations to justifying future developments and applications in VIS to MIR spectroscopy of small bodies in the planetary system.

\section{$\underline{\text { Rosetta and comet } 67 P}$}

VIRTIS on board Rosetta provided $0.25-5.1 \mu \mathrm{m}$ spectra of 67P's surface ${ }^{11,17,19}$. VIRTIS combined three data channels in one compact instrument. Two of the spectral channels were dedicated to spectral mapping (-M) in the range from 0.25 to $5.1 \mu \mathrm{m}(\mathrm{IFOV}=250 \mu \mathrm{rad} / \mathrm{pixel}, \mathrm{FOV}=64 \times 64 \mathrm{mrad})$. The third channel was devoted to high resolution spectroscopy $(-$ H) between 2 and $5 \mu \mathrm{m}$ (spectral sampling 0-6 $-1.6 \mathrm{~nm}, \mathrm{FOV}=0.44 \times 1.34 \mathrm{mrad}$ ). The spectral sampling of VIRTIS-M was $1.8 \mathrm{~nm} / \mathrm{band}$ shortward of $1 \mu \mathrm{m}$ and $9.8 \mathrm{~nm} / \mathrm{b}$ and between 1 and $5 \mu \mathrm{m}$, while for VIRTIS- $\mathrm{H} \lambda / \Delta \lambda$ varies between 1300 and 3000 in the $2-5 \mu \mathrm{m}$ range.
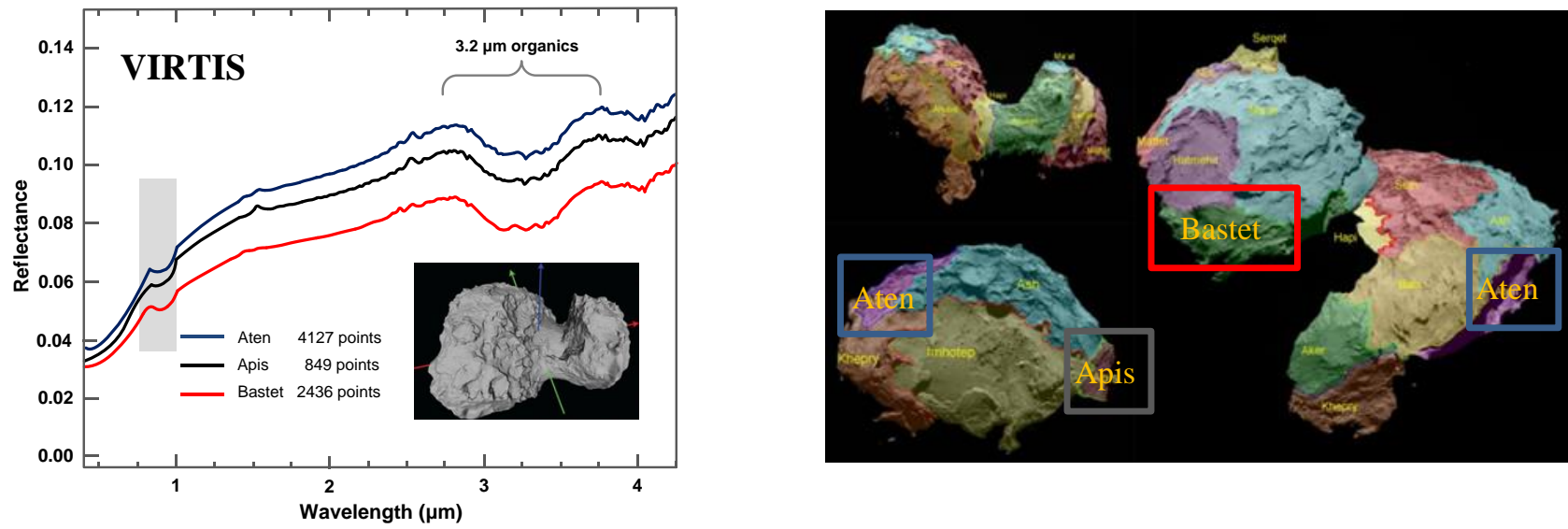

Figure 1. Left: Average VIRTIS-M reflectance spectra ${ }^{6}$ of three morphologic units in the 0.5-4.2 $\mu$ m range: Aten (blue), Apis (black), and Bastet (red) adapted from Filacchione et.al. ${ }^{19}$; gray rectangle left: VIS-IR connection range affected by low signal-to-noise conditions. Small image: Shape model of 67P with rotation axis (blue) and equatorial $\mathrm{x}$ and y axis (red, green) ${ }^{20,21,22 .}$

Right: Regional morphologic definitions taken from Thomas et al. (2015) ${ }^{23}$. The areas that correspond to VIRTIS-M spectra shown left are indicated by rectangles of same color. 
Fig. 1 shows selected VIRTIS-M spectra recorded at 67P for different morphologic units. 67P is a comet of the Jupiter family with a perihelion distance of $1.2432 \mathrm{au}$, a semi major axis of $3.463 \mathrm{au}$, an eccentricity of 0.641 , an obliquity of $52^{\circ}$, and rotation period of $12.4304 \mathrm{~h}$ (on May 2015) ${ }^{5}$. Thus, 67P exhibits strong diurnal and seasonal variations. Thermally corrected VIRTIS-M reflectance spectra display a low geometric albedo of 0.06 at $0.55 \mu \mathrm{m}^{18}$, different red VIS and IR spectral slopes, and a broad $3.2 \mu \mathrm{m}$ band.

In context with an overall low geometric albedo the VIRTIS-M spectra are consistent with refractory organics mixed with opaque minerals, e.g. FeS. Organic $\mathrm{OH}, \mathrm{COOH}$, aliphatic and aromatic $\mathrm{C}-\mathrm{H}$ and $\mathrm{NH}_{4}$ might contribute to the $3.2 \mu \mathrm{m}$ band ${ }^{24}$. In addition, activity driven water ice exposures of various grain sizes could be unambiguously detected and modeled on the basis of the VIRTIS-M NIR spectra ${ }^{25}$. Thermal evolution of complex hydrocarbon materials leads to the decrease of NIR spectral slopes, IR reflectance, and absorption band depths ${ }^{24,}{ }^{26}$. In addition, fine-grained opaque materials intimately mixed with other surface components can significantly contribute to suppress the absorption bands of these components and in extreme cases completely suppress them ${ }^{26}$.

Although they could not be detected directly in this spectral range anhydrous silicates are plausible candidates that one would expect in cometary material. The evidence and the nature of these components would provide important further insights into the development history of comets and pointers to their place of origin and their thermal development. The most promising way to detect such components that cannot be detected in the VIS/NIR spectral range is spectral analysis in the thermal IR.

\section{Rosetta and asteroids 2867 Śteins and 21 Lutetia}

Śteins: Śteins was observed by Rosetta during its flyby in 2008. The VIS/NIR spectrum of 2867 Steins derived between 0.25 and $0.9 \mu \mathrm{m}$ from 11 filters of Rosetta's scientific camera OSIRIS WAC (Optical, Spectroscopic, and Infrared Remote Imaging System, Wide Angle Camera) and 9 filters from the OSIRIS NAC (Narrow Angle Camera) is slightly reddish. It shows a steep drop in the wavelength range shortward of $0.4 \mu \mathrm{m}$ and a characteristic absorption feature at 0.49 $\mu \mathrm{m}$, tentatively attributed to sulfides ${ }^{27}$. VIRTIS-M VIS spectra of Šteins are characterized by a red slope in the visible range as well and by featureless NIR spectra ${ }^{28}$. Thus, the surface mineralogy of Šteins is dominated by iron-poor/free silicates. These spectral properties and a relatively high albedo are compatible with that of an E-type asteroid. E-Type asteroids are thought to be parent bodies of aubrite meteorites. Such meteorites are highly reduced achondrites, composed of iron-poor and magnesium-rich silicates mainly enstatite, with various proportions of diopside, inverted pigeonite, forsterite, albitic plagioclase, as well as small amounts of troilite and metallic Fe, N,i and they may host rare accessory sulfides like oldhamite $(\mathrm{CaS})^{29}$. Aubrites are breccia of igneous cumulates that have been formed in an environment with temperatures above $1000^{\circ} \mathrm{C}$. Steins is therefore probably a body that originates from the interior of a larger and more differentiated basic body ${ }^{30}$.

Lutetia: Fig. 2 (a) shows the VIRTIS-M IR radiance factor (I/F, where I is the measured radiance and F is the solar flux) between 1.0 and $5.1 \mu \mathrm{m}$, which was recorded during the fly-by of the main belt asteroid 21 Lutetia in $2010^{31}$. I/F values increase longward of $4 \mu \mathrm{m}$. This is caused by thermal emission, which begins above $4 \mu \mathrm{m}$ to contribute to the signal and increases with increasing wavelength in the VIRTIS range. In general, the I/F spectrum is featureless. The asteroid spectrum has a moderate red slope in the 0.5 to $0.8 \mu \mathrm{m}$ range, whereas it is flat in the IR range. VIRTIS identified neither absorption bands of hydrated minerals nor indications of organic material. The spectra are mostly uniform over the whole surface of the asteroid. The low thermal inertia derived $\left(<50 \mathrm{Jm}^{-2} \mathrm{~s}^{-0.5} \mathrm{~K}^{-1}\right)$ is characteristic for a lunar like regolith texture ${ }^{31}$. The relatively high mean density of Lutetia ${ }^{32}$ of $3.4 \mathrm{~g} / \mathrm{cm}^{3}$ and the observed spectra indicate that Lutetia is probably less differentiated and a remnant of an original planetary population. It could be composed of chondritic materials dominated by iron-poor minerals that have not undergone aqueous alteration ${ }^{31}$.

Fig. 2 (b, c) show the normalized UV to NIR reflectance spectrum (top) and the mid-infrared (MIR) emittance spectrum of 21 Lutetia data from the OSIRIS camera onboard Rosetta in the ultraviolet, the NTT (New Technology Telescope; LaSilla Observatory, Chile) in the visible, the IRTF (Infrared Telescope Facility; Mauna Kea, Hawaii) in the nearinfrared, and the Spitzer space telescope in the mid-infrared (taken from Barucci et al. ${ }^{33}$ and Vernazza et al. ${ }^{34}$ ). The reflectance spectrum is in good agreement with the VIRTIS observation. While the reflectance spectrum is largely featureless, the mid-infrared emittance spectrum displays some spectral properties that are characteristic of silicates. 

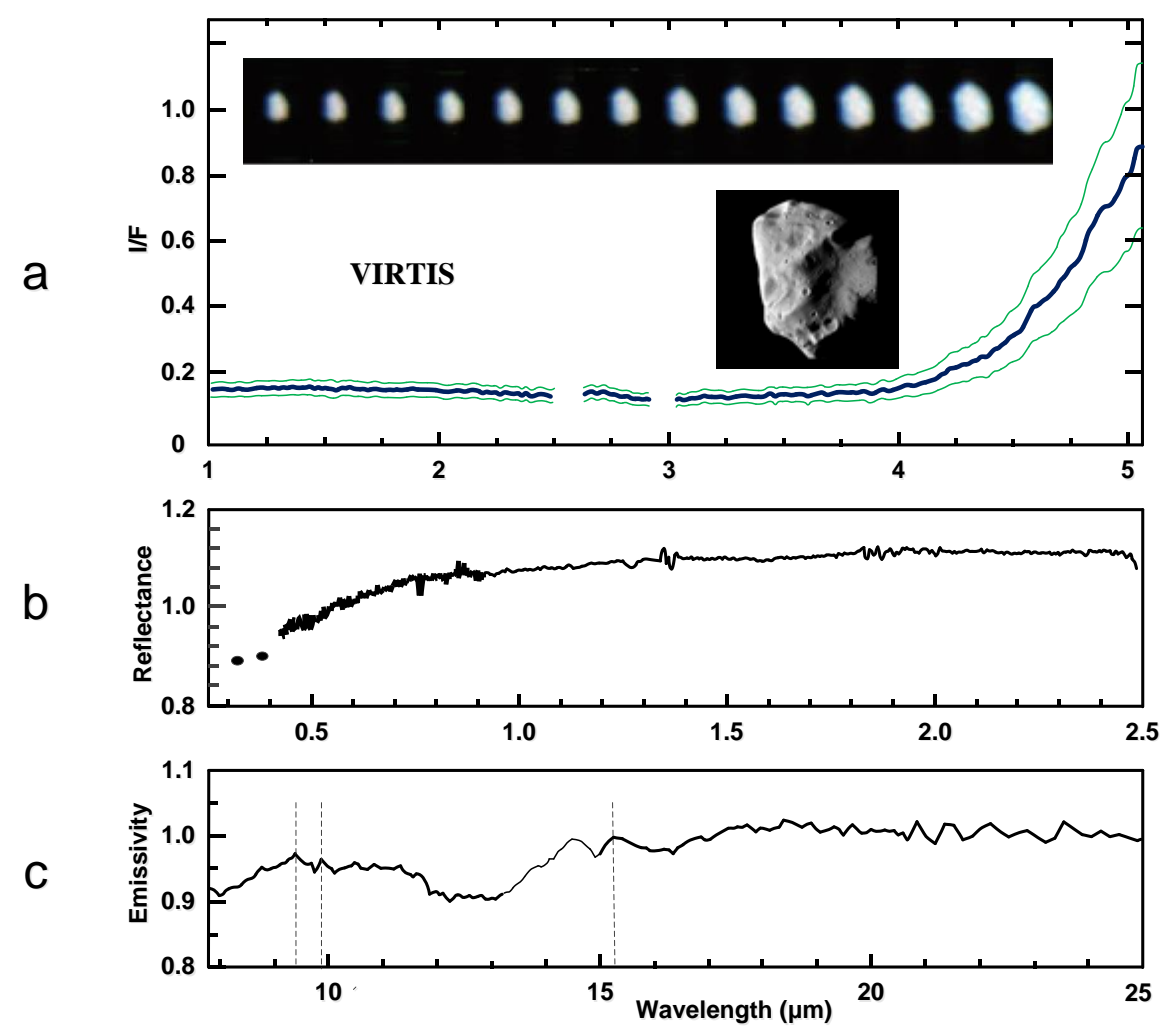

Figure 2. a: VIRTIS-M averaged radiance factor (disk-integrated I/F) of Lutetia taken from Coradini et al. ${ }^{31}$. For each pixel in the upper images a full spectrum was recorded by the instrument. All spectra were averaged to produce the average full disk spectrum in black ( $1 \sigma$ margins - green). Missing ranges in the spectrum correspond to ranges affected by instrumental artifacts. Central image: OSIRIS image of 21 Lutetia $^{32}$.

b, c: Spectroscopic data from Barucci et al. ${ }^{33}$ and Vernazza et al. ${ }^{34}$ combining OSIRIS and ground-based observations: b - UV to NIR reflectance; c - mid-IR emittance (Note: The range between 13.2 and $15 \mu \mathrm{m}$ has measurement problems that are difficult to correct, which is why it is not discussed here); dashed grey lines indicate some relevant spectral features.

The emittance spectrum of 21 Lutetia exhibits emissivity maxima at about $9.4 \mu \mathrm{m}, 9.9 \mu \mathrm{m}$, and $15.3 \mu \mathrm{m}$, a broad transparency band starting from about $12 \mu \mathrm{m}$, and a local emissivity minimum between 15 and $17 \mu \mathrm{m}^{34}$. The fact that these mid-infrared spectral features appear, while the NIR spectra are mostly structure-less, indicates the presence of iron-free to poor and magnesium-rich silicates such as enstatite ${ }^{35}$ and forsterite.

\section{Dawn: main belt asteroid 4 Vesta and dwarf planet 1 Ceres}

In contrast to comets like 67P and smaller asteroids the dwarf planet 1 Ceres and the asteroid 4 Vesta are more differentiated planetary objects. Their greater mass, under the influence of their own gravitation, allowed a greater differentiation, which can be seen in a higher symmetry of their forms. However, their material composition differs considerably. An important reason is the position of their orbits, which for Vesta lies within the frost line of the solar system and for Ceres outside of it. This difference is already manifested in the different mean bulk densities of both objects, which is $3.456 \pm 0.035 \mathrm{~g} / \mathrm{cm}^{-3}$ for 4 Vesta ${ }^{36}$ and $2.161 \pm 0.009 \mathrm{~g} / \mathrm{cm}^{-3}$ for 1 Ceres ${ }^{37}$.

The VIR instrument (Visible and InfraRed spectrometer) operates on the Dawn spacecraft and records reflectance spectra of 4 Vesta and 1 Ceres between 0.25 and $5.1 \mu \mathrm{m}$. As a follow-up development of VIRTIS/Rosetta, VIR has a similar performance like VIRTIS-M, and thus, it is suitable for the analysis of surface composition ${ }^{38}$. 
Vesta: Selected spectra of 4 Vesta ${ }^{39}$ are displayed in Fig. 3. The VIS/NIR spectra are characterized by steep VIS to NIR spectral slopes and absorption bands at 0.9 and around $2 \mu \mathrm{m}$. This clearly indicates that Vesta's soil is rich in unaltered, crystalline orthopyroxenes. The VIR observations confirmed the close correlation of Vesta's surface material with the howardite-eucrite-diogenite (HED) family of basaltic achondrite meteorites. The dominant lithology is howardite-rich in eucrites. Pure diogenites and basaltic eucrites are less abundant at least on the spatial scales resolved by VIR ${ }^{39}$. In addition, the spectra of dark regions show a $2.8-\mu \mathrm{m} \mathrm{OH}$ feature consistent with the presence of hydrated carbonaceous chondrite material in the most ancient regolith. Space weathering to produce nanophase iron particles plays a minor role 40 .
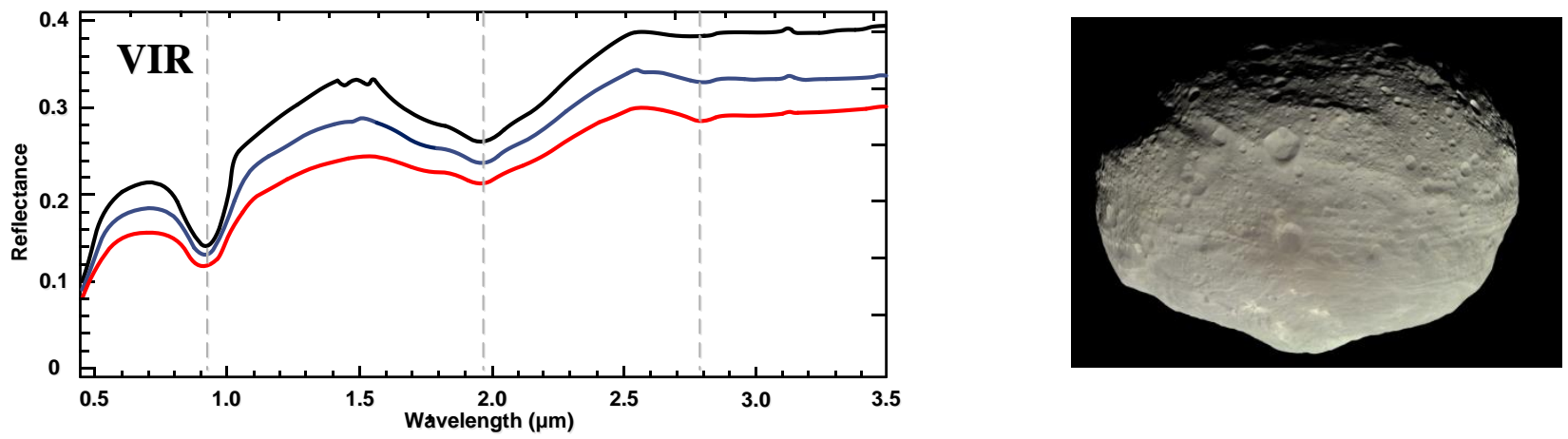

Figure 3. Left: Selected VIR reflectance spectra of 4 Vesta taken from De Sanctis et al. ${ }^{39}$; dashed grey lines indicate absorption bands. Right: 4 Vesta, NASA/JPL-Caltech/UCLA/MPS/DLR/IDA.

Ceres: Ceres' surface shows a broad absorption band between 2.7 and $4.2 \mu \mathrm{m}$ (Fig. 4, left). Within this band several sub bands occur with wavelength positions at $2.72 \mu \mathrm{m}, 3.05-3.1 \mu \mathrm{m}, 3.3-3.5 \mu \mathrm{m}$, and at 3.95 $\mu \mathrm{m}$. These bands have been attributed to magnesium phyllosilicates, ammoniated phyllosilicates, and carbonates, respectively, masked by a dark material ${ }^{41}$. Bright spots like those found in Occator crater display absorption bands at 2.20-2.22 $\mu \mathrm{m}$ and at $2.86 \mu \mathrm{m}$. Spectral near 3.5 and $4.0 \mu \mathrm{m}$ are markedly increased. Thus, the composition of bright spots might be determined by sodium carbonates and ammonia salts ${ }^{42}$. In addition, these bright spots display a blue spectral slope in the VIS-NIR range ${ }^{43}$, and small quantities of ammonium chlorides, bicarbonates, and aluminum clays are needed to account for the 2.20-2.22 $\mu \mathrm{m}_{\text {band }}{ }^{42}$. The nature of the bright material indicates the presence of a volatile-rich subsurface material exposed to the surface either by exogenous processes or recent hydrothermal activity and/or cryovolcanism.
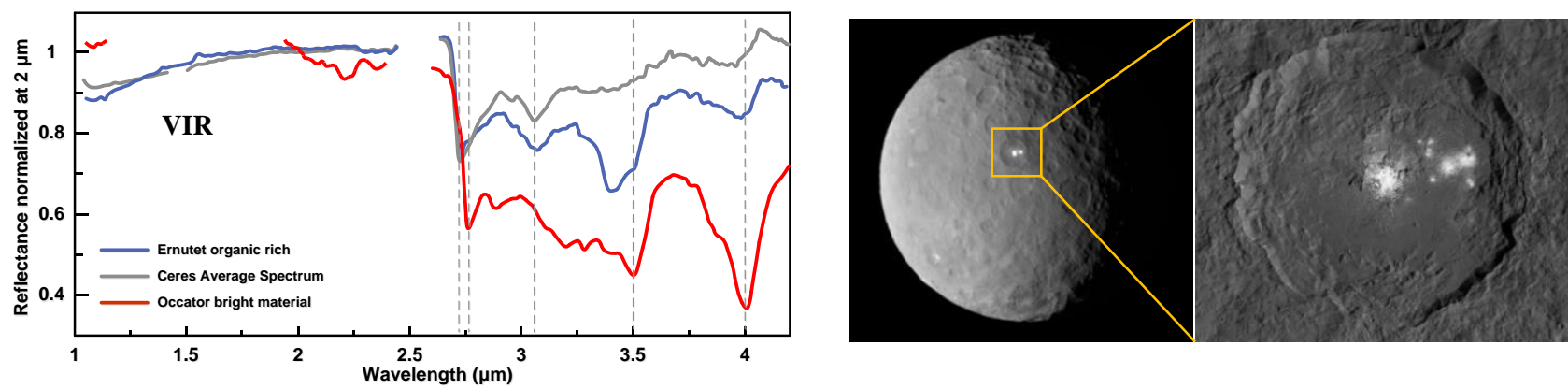

Figure 4. Left: Selected VIR reflectance spectra normalized at $2 \mu \mathrm{m}$ of 1 Ceres taken from De Santis et al. ${ }^{45}$. Middle: 1 Ceres (yellow rectangle: Occator crater, NASA/JPL-Caltech/UCLA/MPS/DLR/IDA).

Right: Occator crater, NASA/JPL-Caltech/UCLA/MPS/DLR/IDA.

The omnipresent distribution of ammoniated phyllosilicates over the entire surface of Ceres suggests a global mixing process by a ubiquitous aqueous alteration ${ }^{44}$. The complex broad absorption between 2.5 and $4.0 \mu \mathrm{m}$ might be influenced locally by refractory organic materials such as aliphatic (and to a certain degree aromatic) hydrocarbons as well ${ }^{45}$. In Fig. 4 an absorption feature at $3.4 \mu \mathrm{m}$ was assigned to aliphatic organic matter in the Ernutet region on Ceres (blue spectrum) 
45. However, the determination of the type of organic material requires a clear separation of the absorption bands from organic and inorganic components, which is an ongoing work..

\section{IMPLICATIONS FOR VIS/IR INSTRUMENT REQUIREMENTS}

Section 2 demonstrates that spectral studies of small bodies require a comprehensive and object-oriented approach to the instrumental technique to be used. Table 1 summarizes some of the most relevant spectral indicators for surface composition analysis. Corresponding to the condensation profile in our solar system volatile and organic components are important constituents of pristine minor bodies in the outer planetary system beyond the frost line.

\section{Spectral indicator}

Low continuum reflectance

High continuum reflectance

Blue or flat VIS-NIR spectral slope

Red VIS-NIR spectral slope

Featureless VIS-NIR spectra

Asymmetric 1- $\mu \mathrm{m}$ absorption band

Absorption bands at 0.9 and between $1.8-2.4 \mu \mathrm{m}$

Absorption bands at $1.05 \mu \mathrm{m}, 1.25 \mu \mathrm{m}, 1.5 \mu \mathrm{m}, 2.0$

$\mu \mathrm{m}$, and $3.0 \mu \mathrm{m}$

Triplet of absorption bands at 1.97, 2.01, $2.07 \mu \mathrm{m}$

and features at 2.7 and $2.78 \mu \mathrm{m}$

Absorption band at about 2.7-2.8 $\mu \mathrm{m}$

Absorption band at about $3.1 \mu \mathrm{m}$

Complex and broad absorption band around 3.2

$\mu \mathrm{m}$

Absorption band at about $4.0 \mu \mathrm{m}$

Emissivity maxima between 7.5 and $9.5 \mu \mathrm{m}$

Broad emissivity absorption band around $10 \mu \mathrm{m}$

Broad emissivity minima between 11 and $15 \mu \mathrm{m}$

Spectral features between 11 and $14 \mu \mathrm{m}$

Multiple absorption bands between 6 and $16 \mu \mathrm{m}$

\section{Possible assignments}

Darkening agents: organic components, dark iron oxides, and fine grained iron sulfides

Iron-free silicates and salts (in combination with corresponding IR absorption bands), water ice deposits in combination with IR absorption bands at $1.05 \mu \mathrm{m}$, $1.25 \mu \mathrm{m}, 1.5 \mu \mathrm{m}, 2.0 \mu \mathrm{m}$, and $3.0 \mu \mathrm{m}$,

Water ice in combination with IR absorption bands at bands at $1.05 \mu \mathrm{m}, 1.25$ $\mu \mathrm{m}, 1.5 \mu \mathrm{m}, 2.0 \mu \mathrm{m}$, and $3.0 \mu \mathrm{m}$, iron-poor salts and phyllosilicates

Iron-rich minerals, nanophase iron produced by space weathering, Fe-sulfides, refractory organic components (in combination with broad absorption bands near $3.2 \mu \mathrm{m}$ )

Iron-poor silicates and salts, dark material suppressing absorption bands

Fe containing olivine

Clino- and orthopyroxenes

Water ice

$\mathrm{CO}_{2}$ ice exposed to the surface (e.g. on comet $67 \mathrm{P}^{46}$ )

$\mathrm{OH}$ in phyllosilicates

$\mathrm{NH}_{4}$ in ammoniated phyllosilicates

Refractory organics: aliphatic and aromatic hydrocarbons, solid acids (if applicable mixed with water ice deposits, e.g. on comets)

$\mathrm{CO}_{3}$ in carbonates

Silicates (Christiansen feature)

Silicates (fundamental Si-O vibration band)

Silicates (transparency features)

Refractory organics (CH vibrations)

Solid acids

Table 1. Spectral indicators for minor body surface composition analysis and possible assignments.

Corresponding to the condensation profile in our solar system ices and organic components are important constituents of pristine minor bodies in the outer planetary system beyond the frost line. The study of these materials enables a deeper understanding and tracking of the origin and evolution of these pristine objects. The IR range between 2 and $4 \mu \mathrm{m}$ is suitable for detection and mapping of refractory organic hydrocarbons and solid acids. Often these materials cause complex and broad absorption bands with various contributions of aliphatic and aromatic hydrocarbons to this absorption band. Neighboring spectral signatures of water ice, if present, may be superimposed. This often makes it difficult to clearly assign local reflection minima within these bands. In addition, these spectral features might be significantly suppressed by the presence of dark material decreasing the spectral contrast. The degree of the VIS and IR spectral slope can be used to evaluate the effect of mixtures of refractory organics with dark materials such as fine grained iron sulfides like pyrrhotite and troilite on the spectral properties. Exposed water ice requires the coverage of the wavelength range from 1.0 to $3.2 \mu \mathrm{m}$. The suite of five absorption bands is indicative for water ice. However, the weaker bands can be completely suppressed by dark components. Photometric modeling is required to assign the remaining water ice absorption features to certain quantities of water ice and corresponding ice particle sizes. As far as the water ice enriched areas have a bluer visible spectrum, the visible spectral slope can be used to verify these models. Minerals (silicates, 
salts, sulfides, and possibly oxides/hydroxides, if present) provide another important key for to the body's origin and evolution. For the detection of hydrated minerals like hydrated salts and phyllosilicates, the wavelength range from 2.7 to $3.2 \mu \mathrm{m}$ can be used. In this spectral range the structural $\mathrm{OH}$ complexes are visible. Their strength can be a measure of hydration and indicate aqueous alteration. In the wavelength range from 0.5 to $5 \mu \mathrm{m}$, absorption bands of ferric and ferrous silicates are visible. They enable examining the cosmochemically important pyroxenes and olivine. Although they are highly likely to be present, low-iron non-hydrated silicates like enstatite and forsterite cannot be directly detected in the spectral range between 0.5 and $5 \mu \mathrm{m}$. However, iron-poor/free and magnesium rich silicates are plausible compositional candidates for many minor bodies. These minerals can be detected in the mid-infrared range. The emissivity of minor bodies' soils can be investigated in the mid infrared. This provides information about vibration bands, such as the Christiansen frequency, reststrahlen bands, and transparency features. These spectral bands characterize existing minerals very specifically. For a direct detection of low iron silicates, MIR investigations between 7.5 and $17 \mu \mathrm{m}$ are unavoidable.

Particle size and thermal effects must also be taken into account when analyzing the surface composition. The regolith structure of the surfaces influences the radiative transfer in the soil. The spectral contrast of the diagnostic bands is often reduced for small particles. The surface morphology and topography in combination with variable diurnal and seasonal lighting conditions influences the local temperature and thus the conditions for an absolute emissivity determination. Therefore, good signal-to-noise performance better than 100 is required. On the other hand, the spectral resolution requirements remain moderate (VIS: $1 \mathrm{~nm} /$ spectel, NIR: $10 \mathrm{~nm} /$ spectel, MIR: 10 to $5 \mathrm{~cm}^{-1}$ for interferometers and better than $100 \mathrm{~nm} /$ spectel for imaging spectrometers). Finally, space weathering processes must be taken into account. They are currently much less studied for low iron surface compositions.

\section{CONCLUSION AND OUTLOOK}

Future space missions should target a greater variety of small bodies than before, including the Martian moons Phobos and Deimos, further main belt asteroids, near-Earth asteroids, unvisited Jupiter Trojans and Kuiper belt objects. These will facilitate the analysis of the formation conditions of small bodies at different heliocentric distances. The Hayabusa2 asteroid sample return mission of the Japanese space agency JAXA encountered the near-Earth C asteroid 162173 Ryugu in June 2018 and started an observation program with remote sensing, in situ landing studies (MINERVA II - Micro Nano Experimental Robot Vehicle for the Asteroid mini-lander; MASCOT - Mobile Asteroid Surface Scout) and sample return ${ }^{47}$. The remote sensing complex comprises a near infrared spectrometer (NIRS3). It observes the asteroid at wavelengths of 1.8 to $3.2 \mu \mathrm{m}$ and allows the study of hydrated minerals and other relevant components listed in Table 1 ${ }^{48}$. In addition, the Hayabusa2 remote sensing complex is equipped with the thermal infrared imager (TIR) to investigate the thermophysical properties of the asteroid ${ }^{49}$. The MINERVA II consists of rovers that will be operated on the surface of the asteroid. The rovers house cameras, thermometers, potentiometers, accelerometers, gyro, and photodiodes to explore Ryugu in different locations ${ }^{47}$. MASCOT is a small lander for the investigation of Ryugu. MASCOT's scientific instruments include a multi-spectral radiometer (MASCOT Radiometer, MARA) for measuring radiation flux and IR brightness temperature in six wavelength ranges between 5.5 and $15.5 \mu \mathrm{m}{ }^{51}$ and a near IR hyperspectral microscope (MicrOmega) for in situ characterization of texture and composition of surface minerals in a spectral range of 0.99 to $3.65 \mu \mathrm{m}^{52}$. Finally, an impact sampling system fires a projectile into the asteroids that ejects small grains of material be caught in a sampling horn and brought up into the sampling system to be sealed in containers that will be returned to Earth for comparative analysis of the composition ${ }^{47}$. This approach enables comprehensive texture and composition analysis.

Another sample return mission is OSIRIS-REx (Origins, Spectral Interpretation, Resource Identification, and Security Regolith Explorer, NASA's New Frontiers program) ${ }^{53}$, which will begin to explore the near-Earth C-type asteroid 101955 Bennu in October 2018, after arrival in August 2018. Two optical spectrometers (OVIRS ${ }^{54}$ - OSIRIS-REx Visible and IR spectrometer [0.4 to $4.3 \mu \mathrm{m}$ ] and OTES- OSIRIS-REx Thermal Emission Spectrometer [4 to $50 \mu \mathrm{m}$ ] ${ }^{55}$ ) will map global physical properties, surface structure and composition of the asteroid. A Sample Acquisition and Return Assembly (SARA) will collect samples and return them to Earth in $2023{ }^{53}$. Other space probes to small bodies with spectral instruments in preparation are the Discovery 13 Mission Lucy (NASA) that will flyby four Jupiter $\mathrm{L}_{4}$ Trojans (3548 Eurybates, 15094 Polymele, 11351 Leucus, and 21900 Orus) and the $\mathrm{L}_{5}$ binary Trojan 617 Patroclus ${ }^{56}$. Lucy is equipped with two spectrometers. L'LORRI (Long Range Reconnaissance Imager, Johns Hopkins APL) is a high-spatial 
resolution, visible imager derived from the LORRI instrument on New Horizons ${ }^{57}$. L'TES (Arizona State University) is a thermal infrared spectrometer based on the heritage of OSIRIS-REx OTES. Mission Discovery 14 Psyche will explore the origin of the planetary nuclei by exploring the asteroid 16 Psyche. It is equipped with a multispectral imager. ${ }^{58}$. Missions proposed like the Solar Powered Sail mission, SPS ${ }^{59}$ (Jupiter Trojans, JAXA) and CAESAR ${ }^{60}$ (67P sample return, NASA, New Frontiers program) can perform future explorations of small bodies. Space missions such as Hayabusa2 and OSIRIS-REx and other missions in preparation or planned combine elements of VIS to MIR remote sensing, in-situ spectral/radiometric studies, and sample return analyses. These will provide a ground truth and refine the analysis of spectral remote sensing data, enabling a clear extraction of surface properties such as physical, structural, and compositional properties.

\section{REFERENCES}

[1] Schulz, R., "The Rosetta mission- Exploring solar system formation”, Planet. Space Sci. 66, 1 (2012).

[2] ESA, Rosetta Space Science, http://www.esa.int/Our_Activities/Space_Science/Rosetta (2018).

[3] Arnold, G. E., "Rosetta and 67P/Churyumov-Gerasimenko: a comet under observation", Proc. SPIE 9608, Infrared Remote Sensing and Instrumentation XXIII (1 September 2015), 960802, doi: 10.1117/12.2190445 (2015).

[4] Arnold, G.E. et al., "VIRTIS on Rosetta: a unique technique to observe comet 67P/Churyumov-Gerasimenko - first results and prospects", Proc. SPIE 9608, Infrared Remote Sensing and Instrumentation XXIII, 960803, doi: 10.1117/12.2187208 (2015).

[5] Arnold, G., "67P/Churyumov-Gerasimenko - Rosetta mission shortly before second landing on a comet: a review", Proc, SPIE 9973, Infrared Remote Sensing and Instrumentation XXIV, 99730Q (14 September 2016); doi: 10.1117/12.2237158 (2016).

[6] Arnold G., "Rosetta's studies of comet 67P's surface: a review after the end of the mission", Proc. SPIE 10403, Infrared Remote Sensing and Instrumentation XXV, 104030I (30 August 2017); doi: 10.1117/12.2274747 (2017).

[7] Biele, J. et al., "The landings of Philae and inferences about comet surface mechanical properties", Science 349, aaa9816, doi:10.1126/science.aaa9816.

[8] ESA, Rosetta Orbiter: Instruments, http://www.esa.int/Our_Activities/Space_Science/Rosetta/Orbiter_Instruments (2018).

[9] ESA, Rosetta Lander: Experiment, http://www.esa.int/Our_Activities/Space_Science/Rosetta/Lander_Instruments (2018).

[10] Coradini, A. et al.., "VIRTIS: An imaging spectrometer of the Rosetta mission", Space Science Rev. 128, 529-559 (2007).

[11] Coradini, A. et al., "VIRTIS: An imaging spectrometer for the Rosetta mission", In: Rosetta: ESA's Mission to the Origin of the Solar System, Springer, 565-587 (2009).

[12] Bibring, J.-P. et al., "Mars surface diversity as revealed by the OMEGA/Mars Express observations", Science 307, 1576-1581, doi: 10.1126/science.1108806 (2005).

[13] Drossart, P. et al., "Scientific goal for the observation of Venus by VIRTIS on ESA/Venus Express mission", Planet. Space Sci. 55, 1653-1672, doi: 10.1016/j.pss.2007.01.03 (2007).

[14] Arnold, G.E., "VIRTIS/VEX observations of Venus: overview of selected results", J. Appl. Remote Sensing 6(1) 063580, doi: 10.1117/1.JRS.6.063580 (2012).

[15] De Sanctis, M.C. et al., "The VIR Spectrometer", Space Sci. Rev. 163, 329-369, doi: 10.1007/s11214-010-9668 (2011).

${ }^{[16]}$ Langevin, Y et al., "The MAJIS visible/NIR imaging spectrometer on board the ESA JUICE mission: updated design, implications for performances and science goals", EPSC Abstracts 11, EPSC2017-931-1 (2017).

[17] Capaccioni, F. et al., "The organic-rich surface of comet 67P/ Churyumov-Gerasimenko as seen by VIRTIS/Rosetta", Science 347, 6220, aaa0628-1, doi: 10.1126/science.aaa0628 (2015).

[18] Ciarniello, M. et al., "Photometric properties of comet 67P/Churyumov-Gerasimenko from VIRTIS-M", Astronomy\&Astrophys. 583, A31, doi: 10.1051/0004-6361/201526307 (2015).

${ }^{[19]}$ Filacchione, G. et al., "The global composition of 67P/CG nucleus by Rosetta/VIRTIS. I) Prelanding phase", Icarus 274, 334-349, doi: 10.1016/j.icarus2016.02.055 (2016).

${ }^{[20]}$ Sierks, H. et al., "On the nucleus structure and activity of comet 67P/Churyumov-Gerasimenko", Science 347, 6220, aaa1044-1 (2015).

[21] Jorda, L. et al., "The global shape, density and rotation of Comet 67P/Churyumov-Gerasimenko from preperihelion Rosetta/OSIRIS observations", Icarus 277, 257-278, doi: 10.1016/j.icarus.2016.05.002 (2016).

${ }^{\text {[22] }}$ Preusker, F. et al., "Shape model, reference system definition and cartographic mapping standards for comet 67P/ChuryumovGerasimenko-stereo-photogrammetric analysis of Rosetta/OSIRIS image data", Astron. Astrophys. 583, A33, doi: 10.1051/0004$6361 / 201526349$ (2015).

[23] Thomas, N. et al., "The morphological diversity of comet 67P/Churyumov-Gerasimenko", Science 347, 6220, aaa044-1 (2015).

${ }^{[24]}$ Quirico, E. et al., "Refractory and semi-volatile organics at the surface of comet 67P/Churyumov-Gerasimenko: Insights from VIRTIS/Rosetta imaging spectrometer", Icarus 272, 32-47, doi: 10.1016/j.icarus.2016.02.028 (2016).

${ }^{[25]}$ Filacchione, G. et al., "Exposed water ice on the nucleus of comet 67P/Churyumov-Gerasimenko", Nature 529, 368-372, doi: 10.1038/nature16190 (2016).

[26] Moroz, L.V. et al., "Natural solid bitumens as possible analogs for cometary and asteroid organics: 1 . Reflectance spectroscopy of pure bitumens", Icarus 134, 253-268, doi: 10.1006/icar.1998.5955 (1998). 
[27] Keller, H.U. et al., „E-Type Asteroid (2867) Šteins as Imaged by OSIRIS on Board Rosetta“, Science 327, 190-193, doi: 10.1126/science.1179559 (2009).

[28] Coradini, A. et al., "The VIRTIS observations of 2867 Śteins during the Rosetta fly-by", American Geophys. Union, Fall Meeting 2008, abstract P54B-01 (2008).

[29] Keil, K., "Enstatite achondrite meteorites (aubrites) and the histories of their asteroidal parent bodies", Chem. Erde Geochemistry 70, 295-317, doi: 10.1016/j.chemer.2010.02.002 (2010).

[30] Coradini, A. et al., "VIRTIS observations during Rosetta Flyby of 2867 Š̉teins", EGU 11, 2009-11986 (2009).

[31] Coradini, A. et al., "The surface composition and temperature of asteroid 21 Lutetia as observed by Rosetta/VIRTIS", Science 334, 492-494, doi: 10.1126/scince.1204062 (2011).

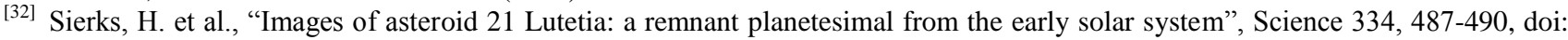
10.1126/science.1207325 (2011).

[33] Barucci, M.A. et al., "Asteroides 2867 S̆teins and 21 Lutetia: Surface composition from far infrared observations with Spitzer space telescope”, Astron. Astrophys. 477, 665-670, doi: 10.1051/0004-6361:20078085 (2008).

[34] Vernazza, P. et al, "Asteroid (21) Lutetia as a remnant of Earth's precursor planetesimals", Icarus 216, 650-659, doi: 10.1016/j.icarus.2011.09.032 (2011).

${ }^{[35]}$ Markus, K. et al., "Reflectance spectra of synthetic Fe-free ortho- and clinoenstatites in the UV/VIS/IR and implications for remote sensing detection of Fe-free pyroxenes on planetary surfaces”, Plant. Space Sci. 159, 43-55, doi: 10.1016/j.pss.2018.04.006 (2018).

${ }^{[36]}$ Russel, C.T. et al., "Dawn at Vesta: testing the protoplanetary paradigm", Science 336, 684-686, doi: 10.1126/science.1219381 (2012).

[37] Raymond, C.A. et al., "Interior evolution of Ceres revealed by Dawn", 47th Annual DPS Meeting, National Harbor, MD, November 2015, 7, 103.01 (2015).

${ }^{[38]}$ De Sanctis, M.C. et al., "The VIR spectrometer", Space Sci. Rev. 163, 329-369, doi: 10.1007/s112114-010-9668-5 (2011).

${ }^{[39]}$ De Sanctis, M.C. et al, "Vesta's mineralogical composition as revealed by the visible and infrared spectrometer on Dawn", Meteoritics \& Planetary Science 48 (11), 2166-2184, doi: 10.1111/maps.12138 (2013).

[40] Pieters, C.M. et al., "The distinctive space weathering on Vesta", Nature 491, 79-81, doi: 10.1038/nature11534 (2012).

${ }^{[41]}$ De Sanctis, M.C. et al., "Ammoniated phyllosilicates with a likely outer solar system origin on (1) Ceres", Nature 528, 241-244, doi: 10.1038/nature16172 (2015).

${ }^{[42]}$ De Sanctis, M.C. et al., "Bright carbonate deposits as evidence of aqueous alteration on (1) Ceres", Nature 536, 54-57, doi: 10.1038 /nature18290 (2016).

[43] Stephan, K. et al., "An investigation of the bluish material on Ceres", Geophys. Res. Lett. 44, 1660-1668, Doi: 10.1002/2016GL071652 (2017).

[44] Ammennito, E. et al, "Distribution of phyllosilicates on the surface of Ceres", Science, 353, 6303, aaf4279, doi: 10.1126/science.aaf4279 (2016).

[45] De Sanctis, M.C. et al., "Localized aliphatic organic material on the surface of Ceres", Science 355, 719-722, doi: 10.1126/science.aaj2305 (2017).

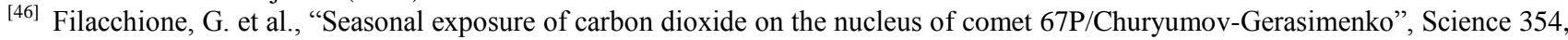
6319, aag3161, 1563-1566, doi 10.1126/science.aag3161 (2016).

[47] Watanabe, S. et al., "Hayabusa2 mission overview", Space Sci. Rev. 208, 3-16, doi: 10.1007/s112114-017-0377-1 (2017).

${ }^{[48]}$ Iwata, T. et al., "The near infrared spectrometer on Hayabusa2", Space Sci. Rev. 208, 317-337, doi: 10.1007/s112114-017-0341-0 (2017).

[49] Okada, T. et al., "The thermal infrared imaging experiments of C-type asteroid 162173 Ryugu", Space Sci. Rev. 208, 255-286, doi: 10.1007/s112114-016-0286-8 (2017).

${ }^{[50]}$ Ho, T.-M. et al., "MASCOT - The mobile asteroid surface scout onboard the hayabusa2 mission", Space Sci. Rev. 208, 339-374, doi: 10.1007/s112114-016-0251-6 (2017).

[51] Grott, M. et al., "The MASCOT radiometer MARA for the Hayabusa2 mission", Space Sci. Rev. 208, 413-431, doi: 10.1007/s112114-016-0272-1 (2017).

[52] Bibring, J.-P. et al., "The MicrOmega investigation onboard Hayabusa2", Space Sci. Rev. 208, 401-412, doi: 10.1007/s11214-0170335-y (2017).

[53] Lauretta, D.S. et al., "OSIRIS-REx: Sample return from Asteroid (101955) Bennu”, Space Sci. Rev. 212, 925-984, doi: 10.1007/s11214-017-0406-1 (2017).

[54] Reuter, D.C. et al., "The OSIRIS-REx Visible and InfraRed Spectrometer (OVIRS): spectral maps of the asteroid Bennu”, Space Sci. Rev. 214, 214:54, doi: 10.1007/s112114-018-0482-9 (2018).

[55] Christensen, P.R. et al., "The OSIRIS-REx Thermal Emission Spectrometer (OTES) instrument", Instrumentation and Methods for Astrophys., arXiv:1704.02390v1 (2017).

${ }^{[56]}$ Levison, H.F. et al., "Lucy: surveying the diversity of the Trojan asteroids: the fossils of planet formation", 48th LPSC, 2025 (2017).

[57] Stanbridge, D. et al., "Lucy: navigating a Jupiter Trojan tour", Preprint AAS 17-632, https://ntrs.nasa.gov/search.jsp?R=20170008008 (2017).

[58] Elkins-Tanton, L.T. et al., "Asteroid (16) Psyche: the science of visiting a metal world", 47th LPSC, 1631 (2016).

[59] Mori, O. et al., "Science experiments on a Jupiter Trojan asteroid in the Solar Powdered Sail mission", 47th LPSC 1822 (2016).

[60] Lauretta, D.S. et al., "The CAESAR New Frontiers mission: 2. sample science”, 49th LPSC, 1334 (2018). 